\title{
Reject Analysis in Conventional Radiography
}

\author{
Usha $^{1}$, Bhargava SK ${ }^{2}$, Bhatt $S^{3}$ \\ ${ }^{1}$ Post Graduate Institute of Medical Sciences, Chandigarh, ${ }^{2}$ School of Medical Sciences and \\ Research, Sharda Hospital, Sharda University, Greater Noida (U.P), ${ }^{3}$ University College of \\ Medical Sciences (Delhi University) \& G.T.B. Hospital, Delhi-110095, India.
}

\begin{abstract}
Quality assurance in diagnostic radiology is of paramount importance to provide quality services leading to better diagnostic yield and thus accurate and timely treatment. Reject analysis study was done in conventional radiography to find out the incidence and the causative factors so that necessary steps be taken to avoid these factors resulting in less repetition of films thus reducing cost and unnecessary radiation to patients and personnel working in radiology department.
\end{abstract}

Keywords: Artifacts, Exposure factors, Conventional Radiography, Dark Room Processing, Positioning.

\section{Introduction}

Plain X-ray Radiographic examination is an integral part of Radiology and Imaging department, even in era where computed and Digital Radiography are gaining importance. Still in the developing countries, about $60 \%$ of the Radiology departments both Government and Private hospitals, small or even bigger are always equipped with conventional X-ray machines for providing basic Radiographic services at the low cost. However, it is not uncommon to encounter poor/suboptimal quality radiographs, leading to repetition of the films thus increasing the cost to excheqor, more radiation to patients'

Corrospondence to: Prof S.K. Bhargava, 201, Fancy Apartment, 19, Vasundhara Enclave, Delhi-110096, India.

E-mail:- skbhargava9731@ @ediffmail.com as well as to the personnel, more time consuming as well as delay in diagnosis to the patient. Hence, it is essential to recognize the factors responsible for diagnostically suboptimal radiographs leading to rejection of the radiographic examination.

World Health Organization (WHO) has recommended a permissible reject rate of $5 \%^{1}$, However, Conference of Radiographic Control Programme Directorate (CRCPD'S) committee on Quality Assurance (QA) recommend a higher reject rate of $10 \% .^{2}$

The purpose of the study was to identify factors responsible for the rejection of conventional radiographs in a Tertiary Care Hospital. 


\section{Material and Method}

Prospective cross-sectional study was carried out in the department of Radiology and Imaging, UCMS \& GTB Hospital thrice a wk. Over a period of 6 months from Dec, 2010-May, 2011 comprising a total of 15388 radiographs. A note was made of the total number of radiographs $(\mathrm{N})$ and total number of reject radiographs (R) in various radiographic examinations (Chest-P.A. view; Skull; PNS; Mandible; Spine lat view; Abdomen; Pelvis and S.I. Joint examination), After finding the causal reject rate in a specific radiographic examination, causal reject for overall exposed radiographs and also for a specific examination was also calculated.

\section{Observation and Results}

Out of 15388 Radiographs, chest constitutes the Maximum Number (48.70\%), followed by extremities (29.20\%), KUB $(8.45 \%)$, Spine $(7.80 \%)$,Skull(5.85\%).Chest accounted for the maximum $(52.64 \%)$ of rejects followed by extremities(26.46\%), together responsible for over $3 / 4^{\text {th }}$ of the rejected radiographs.

Number of the rejected radiographs in a particular radiographic examination was compared with the total number of exposures in that specific examination to find out the reject percentage and found that reject percentage of the spine was the highest (31.41\%), followed by chest $(23.90 \%)$, extremities, KUB and skull was $20.05 \%$, $17.61 \%$ and $11.66 \%$ respectively.

Among the various reasons of rejects, Faulty Exposure constitute the highest (84\%), Selection of KV-Penetration (32\%), faulty positioning of the patient (12.7\%), and rotation $(4.1 \%)$, besides these other factors like collimation, centering, adequacy of the film size were $2.4 \%, 1.08 \%, 0.02 \%$ respectively were within acceptable limits.

Rejection due to various artifacts accounted $51 \%$ out of which the most important were processing related $(24 \%)$, removable artifacts $(18.8 \%)$, faulty film storage and handling accounted for $3.5 \%$ and $4.7 \%$ respectively of the total reasons for rejection. Movement related artifacts were also accounted $0.6 \%$.

\section{Discussion}

Reject analysis is an important part of quality assurance programme in a radiology department providing radiography services to ensure reduction in the factors responsible for rejects and thus to reduce the cost, workload and radiation exposure to patients and personnel.

Out of the total 15388 radiographs exposed, chest X-ray constitutes $-48.70 \%$, extremities$29.10 \%$, spine- $8.45 \%$, KUB-7.80\% and skull $-5.85 \%$ of all exposures. Out of the 15388 radiographs exposed 3404 radiographs were rejected due to one or multiple factors accounting for a reject rate of $22.12 \%$. Zewdeneh D et al. ${ }^{3}$ reported reject rate of $4.94 \%$ for public hospitals and $0.83 \%$ for private hospital which was below the WHO criteria of $5 \% .{ }^{1}$ Hence reject rate in our study is higher than Zewdeneh D et al. ${ }^{3}$ The main reason of discrepancy can be multifactorial like use of old machines, with frequent problem of calibration and collimation, faulty technique used by radiographers due to inattentiveness, excessive workload and not so favorable working environmental conditions can also 
be accountable for the increased reject rate. This further adds various patients' factors like movement, not able to hold breath and non-compliance of instructions by patients were responsible for the rejection of the $\mathrm{x}$ ray films.

For each specific part examined in our study, the percent reject rate of spine radiographs $(31.41 \%)$ was the highest, the percentage reject rate of chest radiographs, extremities, KUB, and skull were- $23.9 \%$, 20.05\%, $17.61 \%$ and $11.66 \%$ respectively. Previous studies ${ }^{3}$ reported the highest rate in chest $\mathrm{X}$ rays $(27.5 \%)$ followed by skull radiographs (25.6\%). Dunn MA et al. $^{4}$ found approximately $50 \%$ of all plain radiographic examination were chest $\mathrm{X}$-rays and had a highest reject rate of $6.5 \%$. In our study, the reject rate in chest and extremities radiographs were due to exposure (mAs) factors and were calculated to be $94 \%$ and $87 \%$ respectively. Similar results were found by Zewdeneh $\mathrm{D}$ et al. $^{3}$ who concluded that highest reject rate is for chest $(27.5 \%)$ and main reason of reject was overexposure (22.8\%) whereas Dunn MA et al. ${ }^{4}$ found positioning as the main cause for reject of the chest radiograph in their study. Both over exposure and underexposure were seen attributed to the performance of the X-ray machines, technical skill and training.

\section{Conclusion}

In order to maintain the good quality radiographs, it is essential to have regular quality assurance programme including regular calibration of the X-ray machines with proper attentiveness of the Technologists to take care factors leading to repetition of the X-ray films and regular morning checking of the processing units.
This cautious approach will definitely reduce the repetition of the films; reduce costs, and unnecessary radiation to patient and personnel working in the Radiology department.

\section{References}

1. Radiation Protection in Diagnostic Radiology, Guidelines, IAEA course material, 2003.

2. Film Use Analysis, Guide lines, CRCPD (Reference in Radiographic Control Programme Directorate), 1986.

3. Zewdeneh D, Tefere S, Admassie D. Xray reject analysis in Tikur Anbessa and Bethzatha Hospitals. Ethiop J Health Dev, 2008;22(1);63-67.

4. Dunn MA, Rogers AT. X-Ray Film reject analysis as a quality indicator Radiography, 1998;4:29-31. 